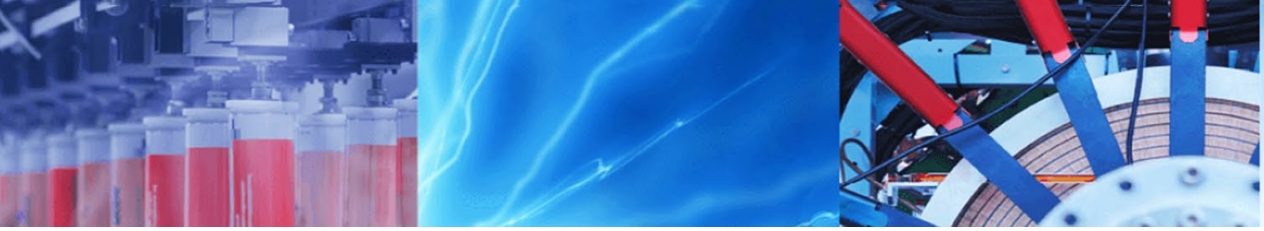

Research Article

\title{
A new segmentation method for non-ideal iris images using morphological reconstruction FCM based on improved DSA
}

\author{
Satish Rapaka' ${ }^{1}$ - P. Rajesh Kumar ${ }^{2} \cdot$ Miranji Katta $^{1} \cdot$ K. Lakshminarayana ${ }^{1} \cdot$ N. Bhupesh Kumar ${ }^{3}$
}

Received: 26 March 2020 / Accepted: 29 December 2020 / Published online: 10 January 2021

(C) The Author(s) 2021 OPEN

\begin{abstract}
In any accurate iris recognition system segmentation of iris plays a vital role. The noise, specular reflections, eyelid/ eyelash obstruction, and intensity inhomogeneities in an image make the segmentation more difficult. In this paper, a novel technique is proposed to segment the iris from images that are taken under uncooperative image conditions. The proposed method segments the image in two stages. Firstly, Morphological reconstruction fuzzy c-means clustering (MRFCM) based on an improved differential search algorithm is implemented before the segmentation step. The MRFCM can preserve image contours even in the presence of noise. Secondly, the iris is isolated from the undesired regions of an eye image by implementing geodesic active contours driven by a modified stopping criterion on the resultant images of the pre-segmentation step. The accuracy of the method presented has been tested on the databases such as CASIAv3-Interval, UBIRISv1, MMU1, IITDv1, and MICHE-I. The segmentation accuracy has been demonstrated and compared with other existing methods present in the literature. The obtained results are promising and the proposed model is outperformed the existing methods.
\end{abstract}

Keywords Iris segmentation · FCM clustering · Morphological reconstruction · Differential search algorithm · Geodesic active contours

\section{Introduction}

The individual identification based on the iris is one of the most important biometrics due to its unique and apparently stable iris patterns. The noise artifacts such as eyelashes/eyelids occlusion, specular reflections, blurring, non-circular iris boundaries, off-axis gaze, etc., make the segmentation more difficult. The segmentation algorithms can be classified broadly into two groups. Segmentation of perfect (ideal) iris images and segmentation of degraded (non-ideal) iris images. For the segmentation of ideal iris images, numerous techniques have been suggested by many researchers in the literature [1-9]. Segmentation of degraded iris images has grabbed attention for a decade.
Even though numerous approaches have been suggested for the segmentation of non-ideal iris image [10-18] there is still room for improvement in robustness and efficiency.

A wide range of segmentation approaches can be found in the literature, which include level set model [15], active contours [16], clustering [19, 20], watershed transform [21], graph cut [22], region growing [23], deep learning [24], etc. Among these varieties, clustering is employed for segmentation due to its rapidity and effectiveness. The purpose of clustering is to group the pixels of an image into various subgroups, based on pixel intensities, which are called clusters. Pixel intensities that belong to the same subgroup are as similar as possible to each other, whereas adjacent groups share the maximum difference.

$\triangle$ Satish Rapaka, rsatishus@gmail.com; N. Bhupesh Kumar, bhupesh.eee.crr@gmail.com | ${ }^{1}$ Department of Electronics and Communication Engineering, Sir C. R. Reddy College of Engineering, Eluru, Andhra Pradesh 534 007, India. ${ }^{2}$ Department of Electronics and Communication Engineering, Andhra University College of Engineering (A), Andhra University, Visakhapatnam, Andhra Pradesh 530003 , India. ${ }^{3}$ Department of Electrical and Electronics Engineering, Sir C. R. Reddy College of Engineering, Eluru, Andhra Pradesh 534 007, India. 
One popular clustering algorithm used widely to solve the segmentation problem is the Fuzzy c-means (FCM) algorithm. It is a soft clustering method, which allows image pixel intensities belong to more than one subgroup and thus has a better ability to tolerate disturbance resulting from noisy data $[19,25,26]$.

However, the conventional FCM has the following shortfalls: (1) It is essentially a local search optimization algorithm, therefore sensitive to initialize the initial cluster centers $[27,28]$. To address this problem, many heuristic approaches have been proposed by many researchers. For instance, a genetic algorithm (GA) based clustering method has been introduced in [29]. Particle swarm optimization based FCM methods have been employed in [30]. The authors in, [31-33] proposed an image segmentation algorithm using an improved form of the differential evolution (DE) algorithm based FCM. Recently, authors in [34], proposed a forest optimization algorithm based FCM to obtain optimized cluster centers. (2) FCM is based on graylevel information hence, fails to segment complex textured and noisy images. To address this, authors have been proposed algorithms which use the local spacial information of the image [35-38]. These techniques are implemented by considering intensity inhomogeneities, employing median and average filtering to obtain spacial neighborhood information and grayscale histograms instead of pixels. However, they do not guarantee noise immunity and preservation of image details. The authors in [39], proposed fast \& robust FCM (FRFCM) which employs morphological reconstruction (MR) filters to guarantee both noise immunity and preserving of the image details. Motivated by this idea, in our early work [40], proposed an improved particle swarm optimization based morphological reconstruct fuzzy c-means (IPSO-MRFCM) to segment the degraded iris images in order to improve the segmentation efficiency.

In this work, another efficient method has been presented for the segmentation of iris images in the iris recognition framework ${ }^{1}$ (Fig. 1) in order to enhance the efficiency further. Our main contributions to this work are:

- The proposed method employs an improved differential search algorithm (IDSA) based morphological reconstruction FCM to smooth the image and to highlight the contours of the objects present in an image. The optimization method IDSA is employed to keep away FCM trapped into a local optimum and lead to the global optimum.

\footnotetext{
1 IDS-MRFCM: Improved differential search algorithm based morphological reconstruct fuzzy c-means,

GACs: Geodesic Active Contours.
}

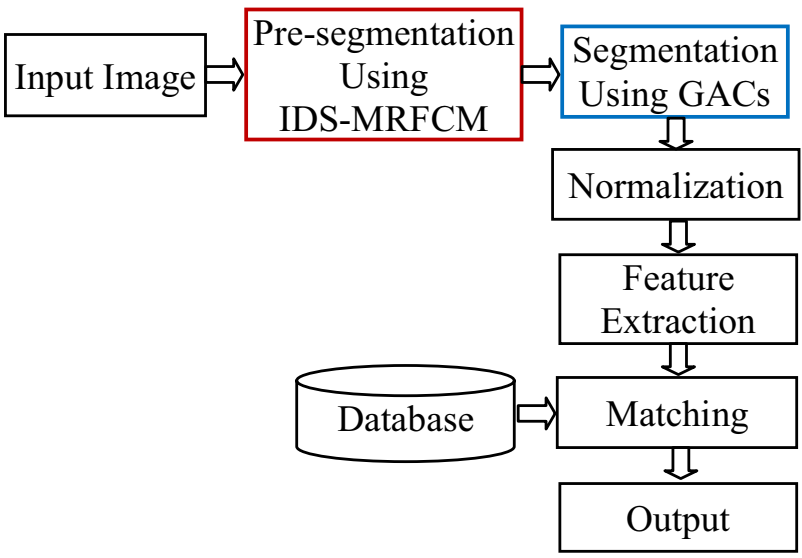

Fig. 1 The block diagram representation of the proposed method

- The isolated iris region is then separated from the unwanted regions by applying geodesic active contours (GACs) incorporated by a modified stopping criterion. Therefore, the proposed method is more robust than the existing methods for non-ideal images.

The rest of the paper is organized as follows. Section 2 provides the details of some baseline segmentation methods. The proposed method has been described in Sect. 3 . Experimental results are discussed in Sect. 4. Finally, the paper has been concluded in Sect. 5 .

\section{Baseline methods for iris segmentation}

\subsection{Integro-Differential Operator}

Baseline segmentation methods are first defined, to demonstrate the proposed method performance improvement. A variant of Hough transform, an Integro-differential operator(IDO), has been used by Daugman in [1, 2], which detects a circular boundary in an edge mapped image.

$\max _{\left(r, x_{i p}, y_{i p}\right)}\left|\frac{\partial}{\partial r} G_{\sigma}(r) * \oint \frac{I(x, y)}{2 \pi r} d s\right|$

In Eq. (1), $r$ and $\left(x_{i p} y_{i p}\right)$ describes the radius and center of the iris or pupil, respectively. $I(x, y)$ is the image to be segmented and $G_{\sigma}(r)$ is a Gaussian filter. The IDO searches iteratively such that the difference in radial image pixel intensity is maximum.

\subsection{Geodesic active contour (GAC)}

The GAC is a combination of energy functional minimization and geometric model $[18,41]$. Initially, the stopping 
function $K$ of an image to be segmented is determined by using Eq. (5). Here the images to be segmented are presegmented images. To avoid the termination of evolving GAC curve due to the edges of the pupil and the local minima formed as a result of thick radial furrows, dark crypts, and specular reflections a modified stopping function $K^{\prime}$ (Eq. (4)) used in [20], has been employed.

A contour with a radius of $r_{p}+5$, where $r_{p}$ is the pupil radius, which is initialized as the initial contour near the pupil. The embedding function $\varphi$ is evolved iteratively according to Eq. (2) until it meets the stopping criterion.

$\varphi_{i, j}^{t+1}=\varphi_{i, j}^{t}+\Delta t\left(-c K_{i, j}^{\prime}\left\|\nabla \varphi^{t}\right\|-K_{i, j}^{\prime}\left(\varepsilon \varphi_{i, j}^{t}\left\|\nabla \varphi^{t}\right\|\right)+\nabla \varphi_{i, j}^{t} . \nabla K_{i, j}^{\prime t}\right)$

$\phi=-\frac{\varphi_{x x} \varphi_{y}^{2}-2 \varphi_{x} \varphi_{y} \varphi_{x y}+\varphi_{y y} \varphi_{x}^{2}}{\left(\varphi_{x}^{2}+\varphi_{y}^{2}\right)^{\frac{3}{2}}}$

where $\phi$ is the curvature computed by considering current level set values, $\varphi$ is the gradient of the image. For an effective implementation time step $\Delta t=0.05$, velocity of evolution $c=0.65$, and degree of smoothness $\varepsilon=1$ are chosen, which are suggested by Shah \& Ross in [18].

$K^{\prime}=K+\delta_{g}^{(n)}(f)$

where

$K(x, y)=\frac{1}{1+\left(\frac{\|\nabla(G(x, y) * l(x, y))\|}{\beta}\right)^{\alpha}}$

and

$\delta_{g}^{(n)}(f)=\delta_{g}^{(1)}\left(\delta_{g}^{(n-1)}(f)\right)$

with

$\delta_{g}^{(1)}(f)=(f \oplus b) \cap g$

In Eq. (4), $\delta_{g}^{(n)}(f)$ is the dilation operator, structuring element $b$ of size $3 \times 3$ is chosen. ' $g$ ' is the marker image, and the mask image is denoted by $f$. In Eq. (5), ' $l$ ' is an image to be segmented, ' $G$ ' is the Gaussian kernel, a and $\beta$ are constants.

\section{The proposed method}

\subsection{Morphological reconstruction FCM Algorithm}

A fuzzy clustering algorithm is an approach used for grouping the pixels of an image into different subgroups based on their attribute features similarities. Image pixels with the same attributes belong to the same subgroup. Fuzzy C-means (FCM) method is one of the prominent fuzzy clustering algorithms often used in many data segmentation applications $[19,30]$. In the proposed MRFCM algorithm similarity measure is determined by using Euclidean distance. The fitness function to be minimized is described as follows.

$J_{M R F C M}=\sum_{k=1}^{n} \sum_{i=1}^{c} u_{i k}^{q}\left\|\tilde{x}_{k}-v_{i}\right\|^{2}$

where, $\tilde{x}$ is a morphological reconstructed image is defined as follows:

$\tilde{x}=R^{O C}\left(f^{\prime}\right)$

where $R^{O C}$ denotes morphological opening-closing by reconstruction (Eq. (14)), and $f^{\prime}$ is the adaptive histogram equalization of the original image.

In Eq. (6), $\left\|\tilde{x}_{k}-v_{i}\right\|$ represents the Euclidean distance between $k$ th data and ith cluster center, $q$ is a real number $(1 \leq q<\infty), u_{i k} \in\{0,1\}$, is the fuzzy membership partition matrix of size $c \times n, c$ is the number of clusters $(2 \leq c<n)$, by updating cluster centers $v_{i}$ (Eq. 8) and membership range $u_{i k}$ (Eq. 9) The fitness function expressed in Eq. (6) is optimized for every iteration, where

$v_{i}=\frac{\sum_{k=1}^{n} u_{i k}^{q} \tilde{x}_{k}}{\sum_{k=1}^{n} u_{i k}^{q}}$

and

$u_{i k}=\frac{1}{\sum_{j=1}^{C}\left[\frac{\left\|\tilde{x}_{k}-v_{i}\right\|}{\left\|\tilde{x}_{k}-v_{j}\right\|}\right]^{\frac{2}{q-1}}}$

Depending on how it differs with the cluster center, a membership value is allotted to a data sample. When the difference is low, a high membership value will be allotted. Iteration process will terminate when the condition $\left\|u_{i k}^{b+1}-u_{i k}^{b}\right\|<\epsilon$ is satisfied, where $b$ is the number of iterations.

\subsection{Morphological opening-closing by reconstruction}

Distribution characteristics of data are sensitive to noise, and hence the FCM is also sensitive to noise [39]. Therefore, results obtained by FCM are not promising for noisy images. This problem is addressed by introducing morphological reconstruction (MR) filters to FCM to optimize the distribution characteristics of data before applying the segmentation using clustering operation. The MR filters 
are capable of preserving iris boundaries and remove unwanted portions of the image. We employ morphological "opening by reconstruction (OBR)" followed by "closing by reconstruction (CBR)" to smoothen the original image.

The MR filters are basically categorized into two. Dilation reconstruction, which meets the condition $g \leq f^{\prime}$, defined by

$R_{f}^{\delta}\left(f_{m}\right)=\delta_{\mu}\left(f_{m}\right)$

and erosion reconstruction, which meets the condition $g \geq f^{\prime}$, defined by

$R_{f}^{\varepsilon}\left(f_{m}\right)=\varepsilon_{\mu}\left(f_{m}\right)$

where $\delta$ and $\varepsilon$ represent dilation and erosion operations, respectively. The combination of Eq. (10) \& Eq. (11) give rise to morphological OBR (Eq. (12)) and CBR (Eq. (13)).

$\gamma_{\mu}\left(f_{m}\right)=\delta_{f}\left(R_{f}^{\varepsilon}\left(f_{m}\right)\right)$

$\varphi_{\mu}\left(f_{m}\right)=\varepsilon_{f}\left(R_{f}^{\delta}\left(f_{m}\right)\right)$

Because morphological OBR followed by $\mathrm{CBR}$, denoted by $R^{O C}$, is more suitable to preserve the boundaries, we employ $R^{O C}$ to modify the original image which is defined by

$R^{O C}\left(f^{\prime}\right)=\varphi_{\mu}\left(\gamma_{\mu}\left(f_{m}\right)\right)$

where $f_{m}$ is the marker image $\left(f_{m}=\varepsilon\left(f^{\prime}\right)\right.$ for OBR and $f_{m}=\delta\left(f^{\prime}\right)$ for (BR) and $f$ is the mask image. In this work, the adaptive histogram equalization of the input image is considered as the mask image.

\subsection{Improved differential search algorithm (IDSA)}

The FCM algorithm is essentially a local search optimization algorithm. To keep away the FCM trapped into a local minimum, a heuristic evolutionary algorithm, the improved variant of the differential search algorithm, has been adopted. Differential search algorithm (DSA) has a better problem-solving ability in global optimization problem [42]. However, its convergence rate is slow in some cases. The convergence rate of the conventional DSA is enhanced in the improved version of the DSA. This is achieved by incorporating the mutation operation of the DE algorithm [43].

In the conventional DSA each individual organisms of a super-organism utilize $N^{*} D$-dimension parameter vector within the bounds $X_{\min }$ and $X_{\max }$ prescribed as follows.

$X_{\text {min }}=\left\{x_{1, \min }, x_{2, \min }, \ldots x_{D, \min }\right\}$
$X_{\max }=\left\{x_{1, \text { max }}, x_{2, \max }, \ldots x_{D, \max }\right\}$

Therefore, the position of individual organisms of an artificial-superorganism is initialized randomly using Eq. (15).

$x_{j, i, 0}=\operatorname{rand}_{i, j} *\left(x_{j, \max }-x_{j, \min }\right)+x_{j, \min }$

where, rand $_{i, j}$ is a arbitrary number distributed uniformly between 0 and 1 . Consider $i=1, \ldots N$ and $j=1, \ldots D$ where, $\mathrm{N}$ refers to the number of discrete individuals in a super-organism and $D$ signifies the size of the problem.

The next step in DSA is to determine the intermediate stopover vectors, $S_{i, G}$ in the migration process, which is expressed by a model called Brownian-like random walk. One of the four forms of $D E$, called $D E /$ rand/1, is used to improve the search mechanism in the improved variant of DSA. The improved variant of DSA is called DS/rand/1. The intermediate stopover site position corresponding to each population individual can be described as follows.

$s_{i, G}=F *\left(X_{r 1, G}-X_{i, G}\right)+X_{r 2, G}$

where, $r_{1}$ and $r_{2}$ are arbitrary integers between $[1,2, \ldots, N]$ and $r_{1} \neq r_{2} \neq i$. $F$ is the scaling factor controls the size of a change in the location of the individuals of the organisms' Eq. (17).

$F=\operatorname{randg}[2 * \operatorname{rand} 1] *(\operatorname{rand} 2-\operatorname{rand} 3)$

where, randg is the gamma random number, and rand1, rand2, and rand 3 are the random numbers uniformly distributed between $[0,1]$.

The stopover site search process can be calculated by the individual of artificial organisms as described in Eq. (18) below.

$s_{i, j, G}^{t}= \begin{cases}s_{i, j, G} & \text { if } r_{i, j}=0 \\ X_{i, j, G} \text { if } r_{i, j}=1\end{cases}$

where, $s_{i, j, G}^{t}$ denotes the trail vector at the $G^{\text {th }}$ iteration.

The next population $(G+1)$, between the stopover site and the artificial-organism population is chosen by using the selection operation described below.

$X_{i, G+1}= \begin{cases}s_{i, G} & \text { if } f\left(s_{i, G}^{t}\right) \leq f\left(X_{i, G}\right) \\ X_{i, G} & \text { if } f\left(s_{i, G}^{t}\right)>f\left(X_{i, G}\right)\end{cases}$

Individuals of the artificial-organisms shift to stopover site, if it is more productive than the origin owned by the artificial-organisms. The artificial-organisms continue its search for the new stopover site from the current position towards the global optimum. 
Table 1 The assigned values for IDS-MRFCM technique

\begin{tabular}{ll}
\hline Parameter details & Parameter values \\
\hline Size of the population $(f)$ & 50 \\
Size of the problem $(D)$ & Number of cluster centers \\
Maximum generations $\left(\mathrm{G}_{\max }\right)$ & 1000 \\
\hline
\end{tabular}

\subsection{The proposed method implementation steps}

The IDS-MRFCM implementation steps have been given below.

Step 1 Set the IDS and MRFCM parameters including population size $(f)$, problem dimension (number of clusters, D), maximum generation count $\left(G_{\max }\right)$ and initial cluster centers (Table 1).

Step 2 Random generation of artificial organisms. Where artificial organisms one position in search space.

LEVELS $=\left[\begin{array}{c}x_{1}^{1} \ldots x_{d}^{1} \\ x_{1}^{2} \ldots x_{d}^{2} \\ \vdots \vdots \vdots \\ x_{1}^{p o p} \ldots x_{d}^{p o p}\end{array}\right]$

$x_{j, i, 0}=\operatorname{rand}_{i, j} *\left(x_{j, \max }-x_{j, \min }\right)+x_{j, \min }$

where, $d$ indicates the number of clusters, $x_{j, i, 0}$ is the value of each individual belonging to the $j$ th cluster center.

Step 3. Compute centroids $\left(v_{i}\right)$, partition matrix (membership degree), $u_{i k}$, according to Eq. (8) \& (9)

Step 4 The objective value of an individual particle is determined from Eq. (6).

Step 5 Update the cluster centers using the IDS algorithm described in the previous section (Eq. 16).

Step 6 Terminate the iteration process if $\left\|u_{i k}^{b+1}-u_{i k}^{b}\right\|<\epsilon$, where $b$ is the number of iterations. Otherwise repeat steps 3-5.

Step 7 Output the optimized $J_{\text {MRFCM }}$.

The implementation flow diagram of IDS-MRFCM has been illustrated in Fig. 2 .

Figure 3 illustrates the implementation of the proposed IDS-MRFCM on a sample image from MICHE-I (IP5) database. Figure $3 \mathrm{a}$ is the input image and Fig. $3 \mathrm{~b}$ is the corresponding grayscale image. Figure $3 c$ shows the presegmented images using conventional FCM, MRFCM and the proposed IDS-MRFCM. Figure 3d indicates the segmented output images as a result of applying GACs (after 490 iterations). It is clearly visible that the proposed segmentation method is successful in isolating the iris from other parts of the input image.

\section{Results and discussion}

The performance of the proposed segmentation method is tested by considering real datasets that are available publicly for researchers. The datasets considered for the experimentation has been presented in Table 2. Four datasets named CASIAv3-Interval, UBIRISv1, MMU1, and IITDV1 have been used for the experiments. The proposed technique is also tested on the MICHE-I database, which was used in the competition named Mobile Iris Challenge Evaluation-I (MICHE-I). Images in the CASIAv3Interval database are pictured under near-infrared (NIR) environments, which is affected by occlusions due to the use of the lens. Images in the UBIRISv 1 and MICHE-I databases are collected under visible wavelength (VW) and uncooperative scenario. The VW images are noisier than the NIR images. Therefore, the segmentation/recognition accuracy of the CASIAv3-Interval and MMU1 is higher than the UBIRISv1 and MICHE-I database.

In the presented method, segmentation / localization is implemented in two stages. The proposed IDS algorithm based MRFCM method (IDS-MRFCM) is employed prior to the segmentation of all the images in the databases (see Fig. 4b). This step isolates the iris region from the unwanted portions of the image. The resultant images are then segmented using GACs driven by a modified termination criterion (see Fig. $4 c-d$ ). The Segmentation performance on some sample images of MMU1, UBIRISv1, and CASIAv3-interval databases have been demonstrated in Figs. 5, 6 and 7, respectively. Figure 5 illustrates the segmentation using the IDS-MRFCM with four cluster centers. Figure $5 \mathrm{a}$ is the input image from the MMU1 database, Fig. $5 \mathrm{~b}$ is the segmented image using the proposed method and Fig. $5 \mathrm{c}$ illustrates the final mask of the iris. The segmentation results are compared with the recent and well existing methods [1, $18,20,40,44,45]$, and are illustrated in Table 3. It has been observed from the obtained results, presented in Table 3, that the proposed method yields good segmentation accuracy compared with the existing techniques.

The segmentation performance of the proposed technique has also been tested on the MICHE-I database. The average image quality of this database is far lower. The images from the MICHE-I database are acquired from different mobile devices like iPhone5 (IP5), Galaxy Samsung IV (GS4), and Galaxy Tablet II (GT2). From each database 480 images (60subjects, 8samples each), which consists of images captured from front and back cameras, indoor and outdoor acquisitions, are taken for the experimentation of the method proposed. Figure 8 demonstrates the segmentation of sample images from MICHE-I dataset. The segmentation accuracy of the method proposed on 
Fig. 2 Implementation flow diagram of IDS-MRFCM

\section{Start}

1

Read the input image, initialize the IDS algorithm and

FCM parameters including population size $(f)$, problem dimension (number of clusters) $(D)$, maximum generation count $\left(G_{\max }\right)$, initial cluster

Random generation of artificial organisms using Eq. (15)

Compute centroids $\left(v_{i}\right)$, partition matrix (membership degree), $u_{i k}$ according to Eq. (8\&9)

The fitness value of each particle is computed from Eq. (6)

Update the cluster centers using IDS algorithm

Evaluate fitness function using Eq. (6)

$\checkmark$

Compare each solution with corresponding initial solution and find the difference $\left\|u_{i k}^{b+1}-u_{i k}^{b}\right\|$

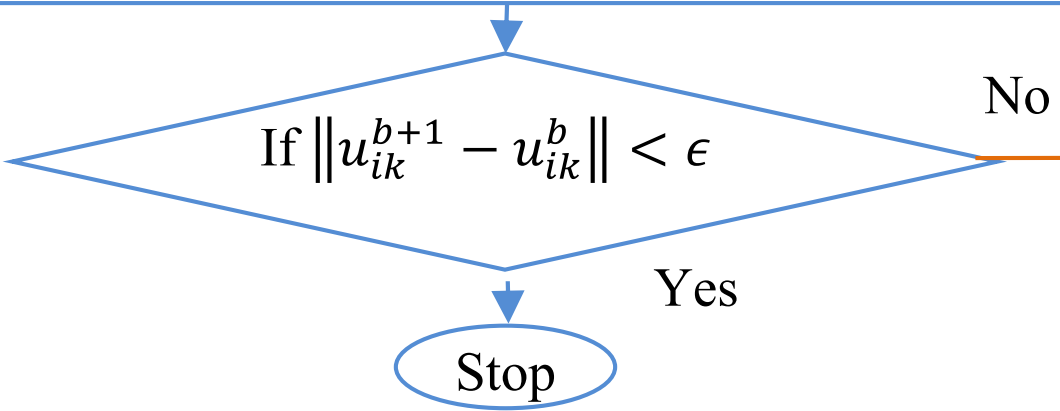




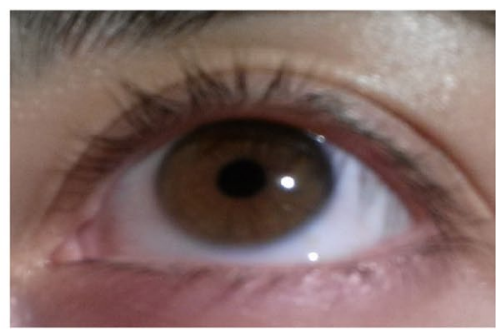

a

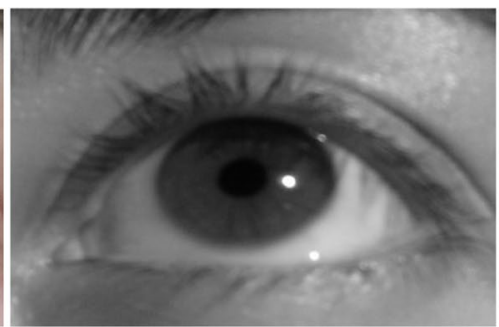

$b$
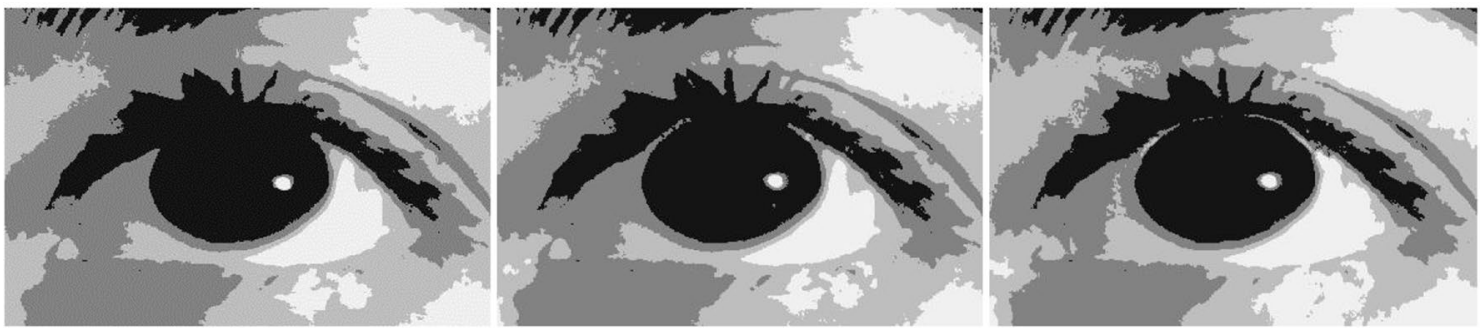

C
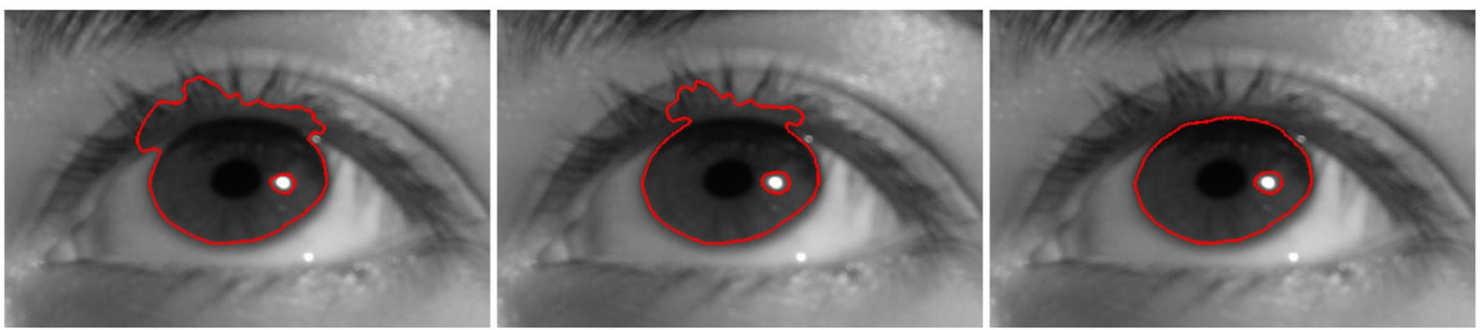

$d$

Fig. 3 Implementation of IDS-MRFCM on a sample image from MICHE-I (IP5) database. a input image, b gray scale input image, $\mathbf{c}$ pre-segmentation using FCM, MRFCM and IDS-MRFCM, respectively, $\mathbf{d}$ segmentation using GACs

Table 2 details of databases

\begin{tabular}{lllll}
\hline Database & Subject & Sample/subject & Total images & $\begin{array}{l}\text { Lightning } \\
\text { conditions }\end{array}$ \\
\hline CASIAv3-interval & $173(\mathrm{~L}), 168(\mathrm{R})$ & 5 each & 1705 & $\mathrm{NIR}$ \\
UBIRISv1 & $241(\mathrm{~s} 1), 131(\mathrm{~s} 2)$ & 5 each & 1860 & $\mathrm{VW}$ \\
MMU1 & $45(\mathrm{~L}), 45(\mathrm{R})$ & 5 each & 450 & $\mathrm{NIR}$ \\
MICHE & 60 & 8 each & 480 & $\mathrm{VW}$ \\
(IP5, GS4, GT2) & & 5 & 500 & $\mathrm{NIR}$ \\
IITDv1 & 100 & 5 & &
\end{tabular}

MICHE-I database has been reported in the Table 4. It is noteworthy that the proposed technique is remarkably effective and yields satisfactory results on MICHE-I database.

The effectiveness of the segmentation method proposed is also tested for the identification/recognition of the system by conducting the post-segmentation process i.e., normalization, feature extraction, and iris code matching. Post-segmentation processing has been carried out using the standard techniques described in [1]. The mean values of hamming distance (HD) distributions for genuine and imposter comparisons have been illustrated in Table 5. The mean of genuine HD distribution against 5115 comparisons from CASIAv3-Interval 


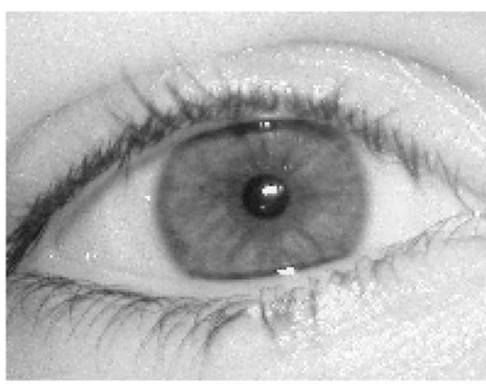

$a$

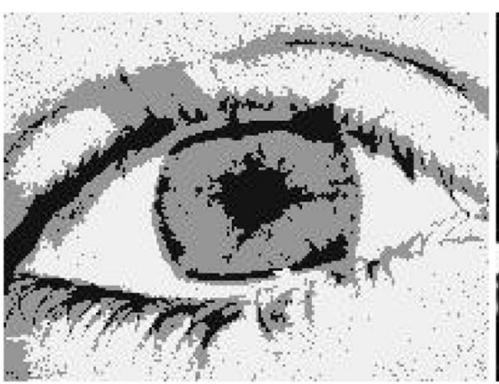

$b$

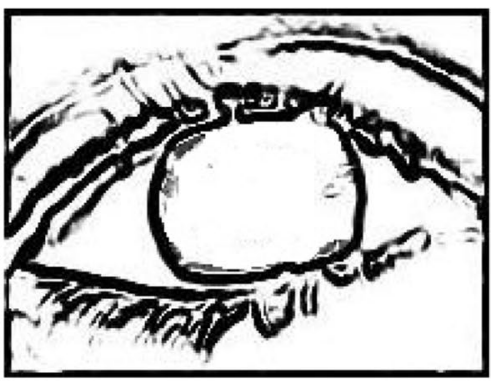

$c$
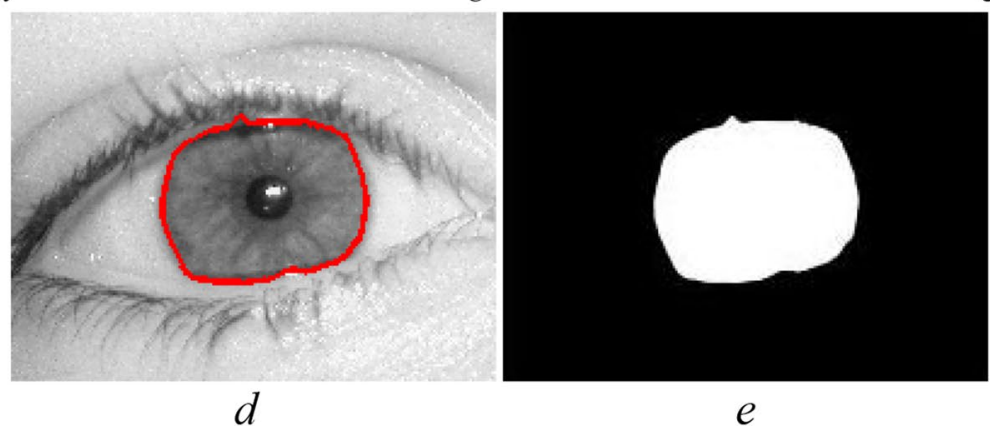

$e$

Fig. 4 The segmentation of a sample image using the proposed method. a Original image, $\mathbf{b}$ pre-segmented image using the proposed IDSMRFCM, c the stopping criterion using Eq. (4), $\mathbf{d}$ segmentation using GACs, e the final mask of the segmented iris
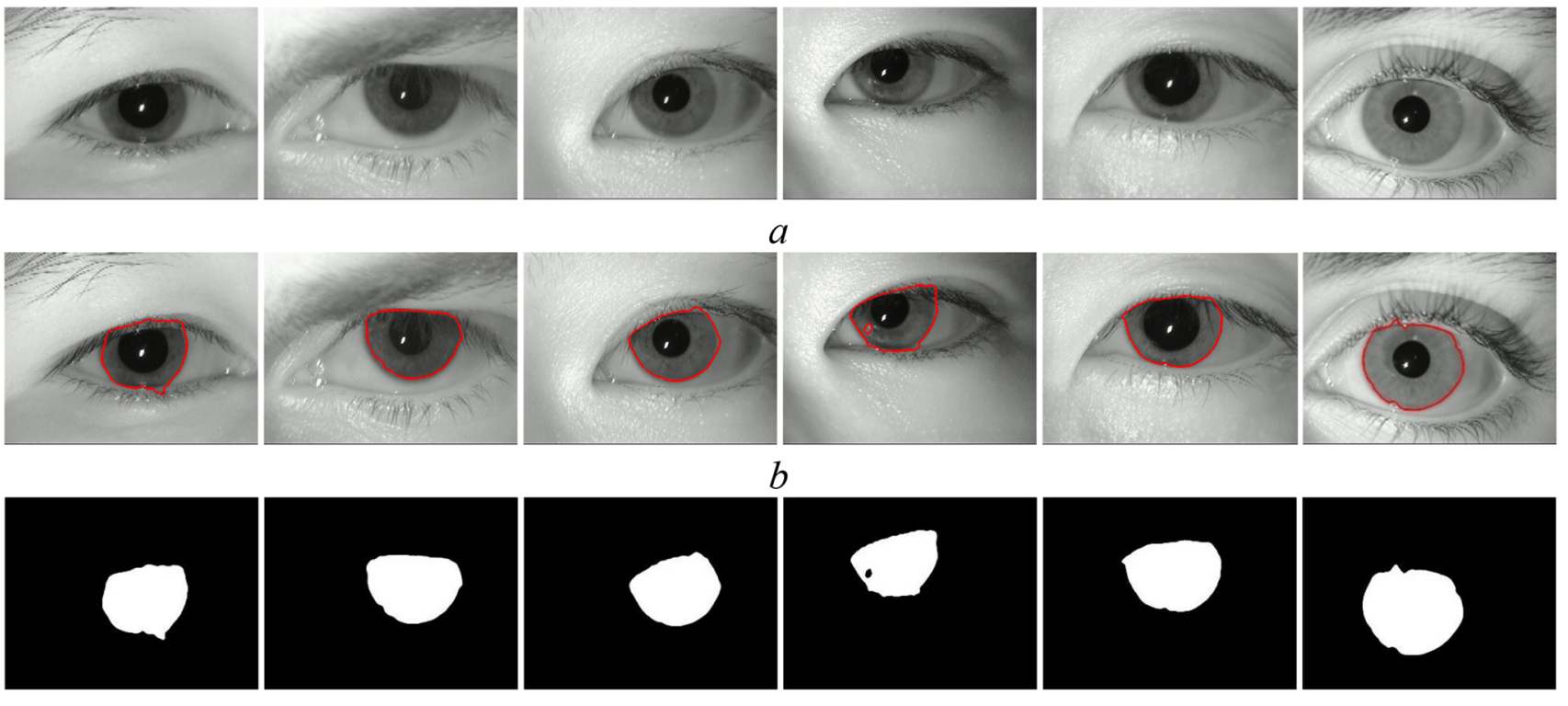

$c$

Fig. 5 Segmentation of iris images from MMU1 database. a Input images, $\mathbf{b}$ segmented output by using the proposed method, $\mathbf{c}$ final mask of the segmentation 

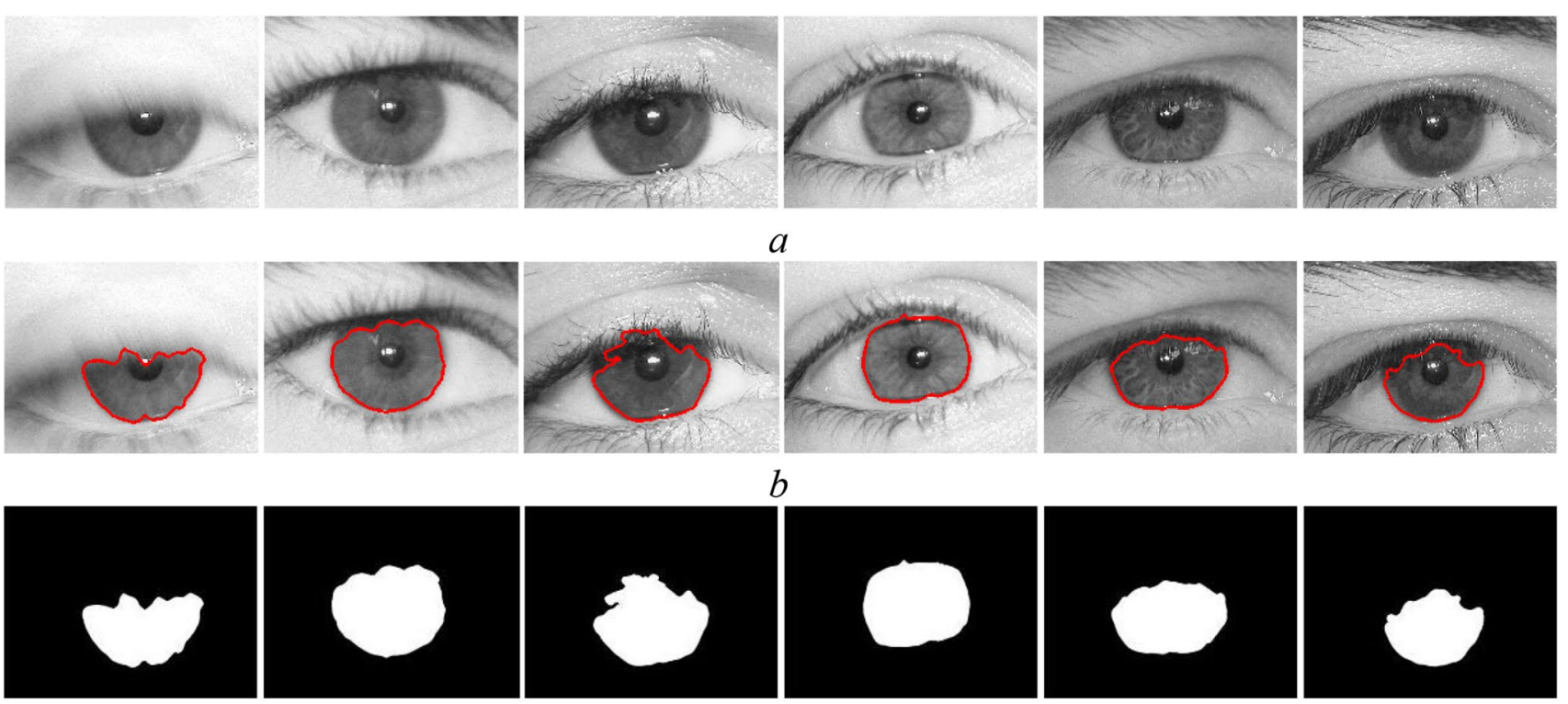

$c$

Fig. 6 Segmentation of iris images from UBIRISv1 database. a Input images, b segmented output by using the proposed method, c final mask of the segmentation
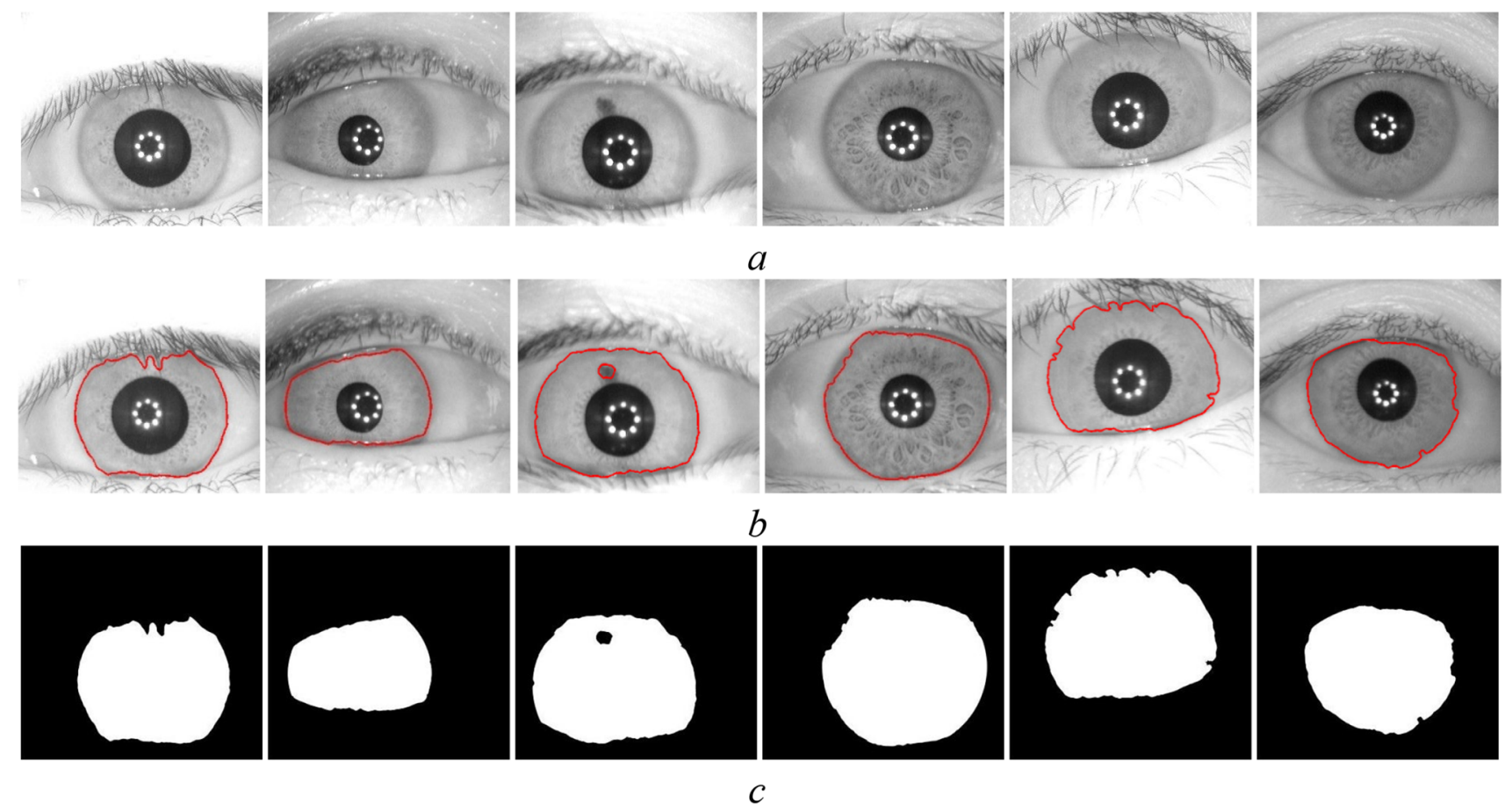

Fig. 7 Segmentation of iris images from CASIAv3-Interval database. a Input images, b segmented output by using the proposed method, c final mask of the segmentation 
Table 3 Segmentation accuracy (\%)

\begin{tabular}{llllc}
\hline methods & CASIA v3 Interval & UBIRISv1 & MMU1 & IITDv1 \\
\hline Proposed & 97.76 & 95.27 & 99.5 & 98.6 \\
IPSO-MRFCM [40] & 96.95 & 93.53 & 98.5 & 98.2 \\
Fuentes-Hurtado et. al [45] & 96.77 & 93.22 & 99.3 & 97.2 \\
PSO-FCM [20] & 95.89 & 92.83 & 99.11 & 96.66 \\
Shah and Ross [18] & 88.56 & 83.3 & 93.77 & - \\
Masek's [44] & 89.97 & 84.4 & 92 & - \\
Daugman's [1] & 59.41 & 52.72 & 75.33 & - \\
\hline
\end{tabular}

Samsung Galaxy S4 database
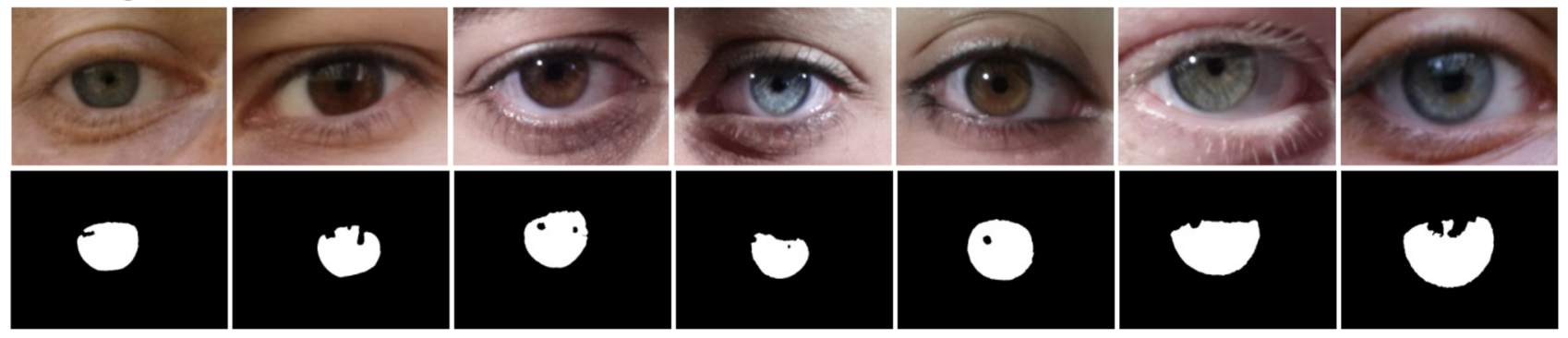

Samsung Galaxy Tab2 database
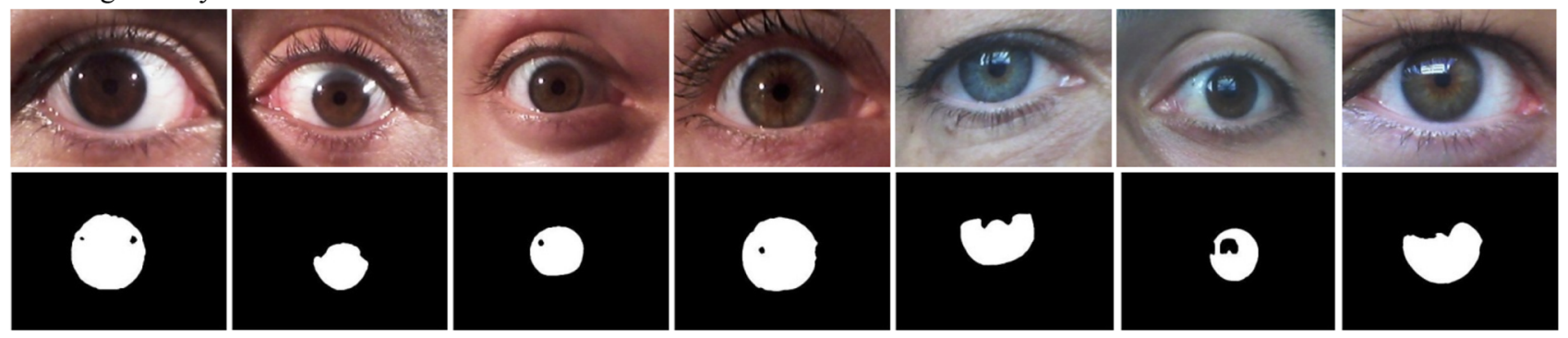

iPhone5 database
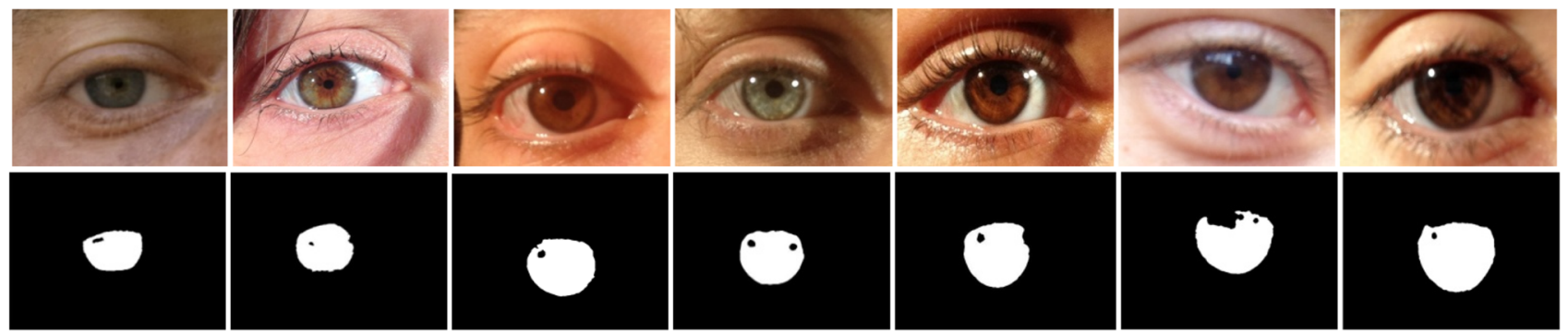

Fig. 8 Segmentation of iris images from MICHE-I database 
Table 4 Segmentation accuracy (\%) for MICHE database

\begin{tabular}{lll}
\hline $\begin{array}{l}\text { MICHE } \\
\text { Database }\end{array}$ & IPSO-MRFCM [40] & $\begin{array}{l}\text { Proposed } \\
\text { IDS-MRFCM }\end{array}$ \\
\hline IP5 & 89.5 & 93.1 \\
GT2 & 88.1 & 92.56 \\
GS4 & 83.9 & 86.43 \\
\hline
\end{tabular}

database is 0.2472 , it is 0.2587 against 5580 comparisons from the UBIRISv1 database, and it is 0.2563 against 1350 comparisons of MMU1 database and is 0.2468 against 1500 comparisons from IITDv1 database. Similarly, the mean of imposter HD distribution against 438,705 comparisons from the CASIAv3-Interval database is 0.4719 and it is 0.4695 against 567,105 comparisons from UBIRISv 1 database. It is 0.4825 against 31,050 comparisons from the MMU1 dataset, and is 0.4820 against 74,250 comparisons for the IITDv1 database. It has been observed that the proposed method yields low HD for genuine comparisons and high HD for imposter comparisons, which is required.

Figure 9, shows the segmentation of some sample images from IITDv1 database. Figure 10, illustrates some sample iris images from MICHE-I database that were not properly segmented. As a result the calculated hamming distance between intra-class samples will be very high, which leads to a wrong rejection.

\section{Conclusions}

The segmentation/localization is a vital stage in any biometric authentication system for better recognition. The noise artifacts added during the capturing of the images under various lighting conditions will lead to improper segmentation. In order to delimit the iris from the other parts of the eye image, a novel morphological reconstructed FCM algorithm based on an improved differential search optimization algorithm has been proposed as a pre-processing step in the iris authentication pipeline. Many segmentation methods relax the assumption that the iris is a non-circle. In this paper, geodesic active contours are employed to segment the non-circular iris boundaries. The obtained results on the CASIAv3-Interval, UBIRISv1, MMU1, IITDv1, and MICHE-I datasets indicate the accuracy of the proposed method. The proposed method achieves high segmentation accuracy when compared with the existing methods. The proposed method can be further extended by incorporating deep learning methods to improve the segmentation accuracy of images with heavily occluded by lightning conditions. Many researchers now a day are implementing deep learning methods in both segmentation and feature extraction stages to improve recognition accuracy in mobile iris recognition applications.

Table 5 Mean of hamming distance (HD) distributions

\begin{tabular}{|c|c|c|c|c|c|c|c|c|}
\hline \multirow[t]{2}{*}{ Method } & \multicolumn{4}{|c|}{ Genuine comparisons } & \multicolumn{4}{|c|}{ Imposter comparisons } \\
\hline & CASIA V3 Interval & MMU1 & UBIRISv1 & IITDv1 & CASIA V3 Interval & MMU1 & UBIRISv1 & IITDv1 \\
\hline Proposed & 0.2472 & 0.2563 & 0.2587 & 0.2468 & 0.4719 & 0.4825 & 0.4695 & 0.4820 \\
\hline IPSO-MRFCM [40] & 0.2510 & 0.2616 & 0.2627 & 0.2613 & 0.4673 & 0.4768 & 0.4648 & 0.4762 \\
\hline Fuentes-Hurtado et. al [45] & 0.2512 & 0.2602 & 0.2635 & 0.2606 & 0.4677 & 0.4775 & 0.4653 & 0.4772 \\
\hline PSO-FCM [20] & 0.2561 & 0.2656 & 0.2680 & 0.2661 & 0.4608 & 0.4688 & 0.4598 & 0.4687 \\
\hline Shah\&Ross [18] & 0.2613 & 0.2696 & 0.2735 & - & 0.4540 & 0.4605 & 0.4543 & - \\
\hline Masek's [44] & 0.2714 & 0.2740 & 0.2821 & - & 0.4500 & 0.4544 & 0.4598 & - \\
\hline Daugman's [1] & 0.2874 & 0.2876 & 0.2885 & - & 0.4466 & 0.4521 & 0.4503 & - \\
\hline
\end{tabular}


Fig. 9 Segmentation of iris images from IITDV1 database. a Input images, b segmented output by using the proposed method, c final mask of the segmentation
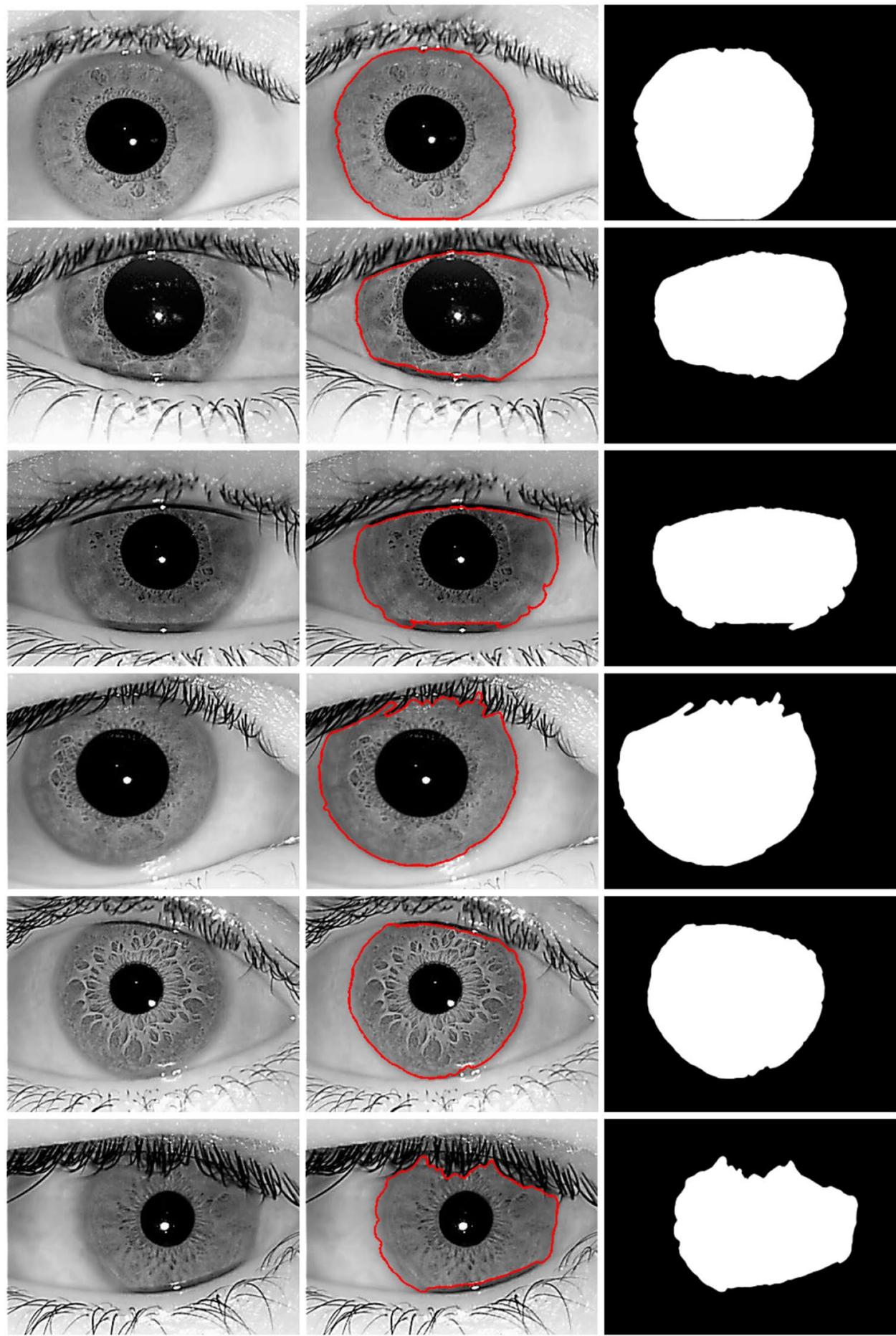

a
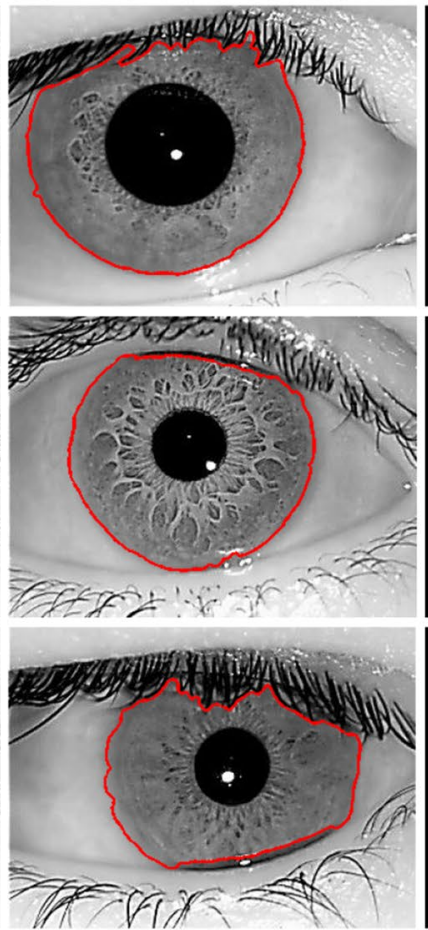

b
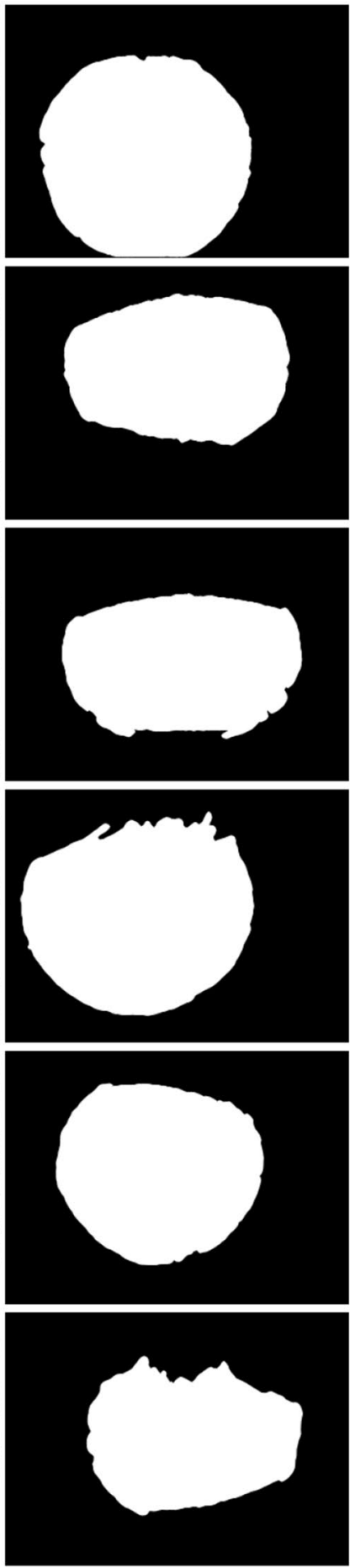

C 
Fig. 10 Some sample images that are wrongly identified. a Input image from MICHEI database, b segmented images, c corresponding masks of the segmented images
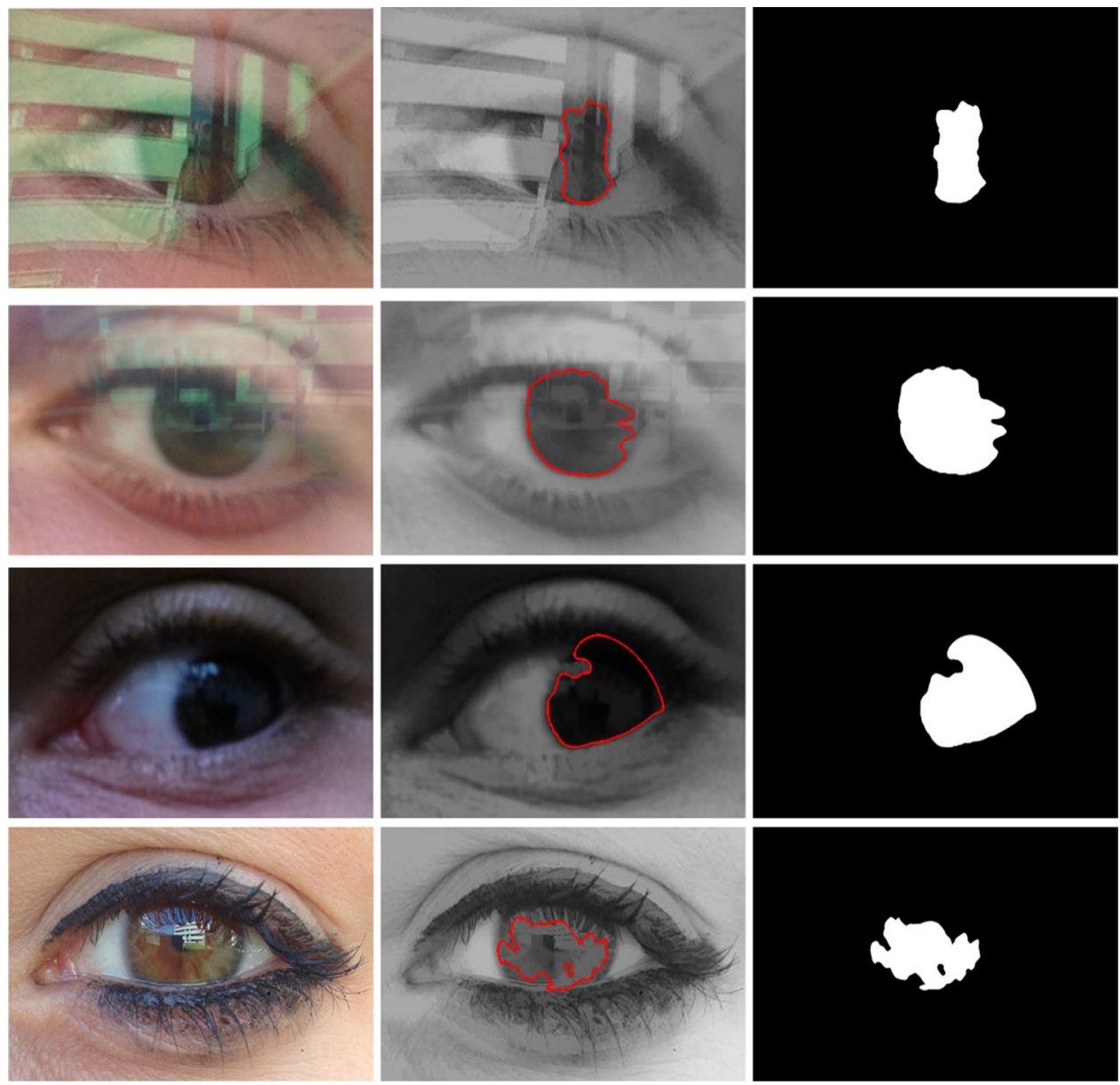

Availability of data and materials The datasets used for the analysis are publicly available online.

Code availability The basic MATLAB codes used to implement the proposed algorithms are available at https://in.mathworks.com/ matlabcentral/fileexchange/5,652, https://in.mathworks.com/matla bcentral/fileexchange/43390, https://in.mathworks.com/help/image $\mathrm{s} / \mathrm{ref} / \mathrm{imreconstruct.html}$ the complete code is available from the corresponding author upon reasonable request

\section{Compliance with ethical standards}

Conflict of interest On behalf of all authors, the corresponding author states that there is no conflict of interest.

Open Access This article is licensed under a Creative Commons Attribution 4.0 International License, which permits use, sharing, adaptation, distribution and reproduction in any medium or format, as long as you give appropriate credit to the original author(s) and the source, provide a link to the Creative Commons licence, and indicate if changes were made. The images or other third party material in this article are included in the article's Creative Commons licence, unless indicated otherwise in a credit line to the material. If material is not included in the article's Creative Commons licence and your intended use is not permitted by statutory regulation or exceeds the permitted use, you will need to obtain permission directly from the copyright holder. To view a copy of this licence, visit http://creativecommons .org/licenses/by/4.0/.

\section{References}

1. Daugman J (2009) How iris recognition works. IEEE Circuits Syst Video Tech 14:21-30. https://doi.org/10.1016/B978-0-12-37445 7-9.00025-1

2. Daugman J (1993) High confidence visual recognition of persons by a test of statistical independence. IEEE Trans Pattern Anal Mach Intell 15:1148-1161. https://doi.org/10.1109/34.244676

3. Ma L, Wang Y, Tan T (2002) Iris recognition using circular symmetric filters. In: Object recognition supported by user interaction for service robots. IEEE Comput. Soc, Quebec City, Que., Canada. Vol 2,pp 414-417. https://doi.org/10.1109/ICPR.2002.1048327.

4. Miyazawa K, Ito K, Aoki T et al (2008) An effective approach for iris recognition using phase-based image matching. IEEE Trans Pattern Anal Mach Intell 30:1741-1756. https://doi.org/10.1109/ TPAMI.2007.70833

5. Ng TW, Tay TL, Khor SW (2010) Iris Recognition using rapid haar wavelet decomposition. In: 2nd international conference signal process systems, pp 820-823

6. Ortiz E, Bowyer KW, Flynn PJ (2016) Dilation-aware enrolment for iris recognition. IET Biom 5:92-99. https://doi.org/10.1049/ iet-bmt.2015.0005 
7. Radman A, Zainal N, Jumari K (2013) Fast and reliable iris segmentation algorithm. IET Image Process 7:42-49. https://doi. org/10.1049/iet-ipr.2012.0452

8. Satish R, Kumar PR (2018) State-of-the art iris segmentation methods: a survey. Int J Comput Sci Eng 6:739-748. https://doi. org/10.26438/ijcse/v6i11.739748

9. Wildes RP (1997) Iris recognition: an emerging biometric technology. Proc IEEE 85:1348-1363. https://doi. org/10.1109/5.628669

10. Rapaka S, Kumar PR (2018) Efficient approach for non-ideal iris segmentation using improved particle swarm optimisationbased multilevel thresholding and geodesic active contours. IET Image Process 12:1721-1729. https://doi.org/10.1049/ iet-ipr.2016.0917

11. Akinfende AS, Imoize AL, Ajose OS (2020) Investigation of iris segmentation techniques using active contours for noncooperative iris recognition. Indones J Electr Eng Comput Sci 19:1275-1286. https://doi.org/10.11591/ijeecs.v19.i3.pp127 $5-1286$

12. Chang Y-T, Shih TK, Li Y-H, Kumara WGCW (2020) Effectiveness evaluation of iris segmentation by using geodesic active contour (GAC). J Supercomput 76:1628-1641. https://doi. org/10.1007/s11227-018-2450-2

13. Fuentes-Hurtado F, Naranjo V, Diego-Mas JA, Alcañiz M (2019) A hybrid method for accurate iris segmentation on at-a-distance visible-wavelength images. EURASIP J Image Video Process 2019:75. https://doi.org/10.1186/s13640-019-0473-0

14. Zhang Q, Li H, Sun Z, Tan T (2018) Deep feature fusion for iris and periocular biometrics on mobile devices. IEEE Trans Inf Forensics Secur 13:2897-2912. https://doi.org/10.1109/TIFS.2018.2833033

15. Malarvizhi N, Selvarani P, Raj P (2020) Adaptive fuzzy genetic algorithm for multi biometric authentication. Multimed Tools Appl 79:9131-9144. https://doi.org/10.1007/s11042-019-7436-4

16. Daugman J (2007) New methods in iris recognition. IEEE Trans Syst Man Cybern Part B Cybern Publ IEEE Syst Man Cybern Soc 37:1167-1175. https://doi.org/10.1109/TSMCB.2007.903540

17. Arsalan $M$, Hong $H$, Naqvi R et al (2017) Deep learning-based iris segmentation for iris recognition in visible light environment. Symmetry 9:263. https://doi.org/10.3390/sym9110263

18. Shah S, Ross A (2009) Iris segmentation using geodesic active contours. IEEE Trans Inf Forensics Secur 4:824-836. https://doi. org/10.1109/TIFS.2009.2033225

19. Cannon RL, Dave JV, Bezdek JC (1986) Efficient implementation of the fuzzy c-means clustering algorithms. IEEE Trans Pattern Anal Mach Intell 8:248-255. https://doi.org/10.1109/ TPAMI.1986.4767778

20. Rapaka S, Kumar Pullakura R (2018) Towards segmentation of non-ideal iris images using optimization based multilevel thresholding. In: 2018 3rd international conference on communication and electronics systems (ICCES). IEEE, Coimbatore, India, pp 46-51. https://doi.org/10.1109/CESYS.2018.8723939.

21. Beucher S, Meyer F (2018) The morphological approach to segmentation: the watershed transformation. In: Dougherty ER (ed) Mathematical morphology in image processing, vol 1. CRC Press, Boca Raton, pp 433-481

22. Pundlik SJ, Woodard DL, Birchfield ST (2008) Non-ideal iris segmentation using graph cuts. In: 2008 IEEE computer society conference on computer vision and pattern recognition workshops. IEEE, Anchorage, AK, USA, pp 1-6. https://doi.org/10.1109/ CVPRW.2008.4563108.

23. Adams $R$, Bischof $L$ (1994) Seeded region growing. IEEE Trans Pattern Anal Mach Intell 16:641-647. https://doi. org/10.1109/34.295913

24. Li Y-H, Huang P-J, Juan Y (2019) An efficient and robust iris segmentation algorithm using deep learning. Mob Inf Syst 2019:1-14. https://doi.org/10.1155/2019/4568929
25. Agrawal S, Vig R (2012) Performance analysis of clustering based image segmentation and optimization methods, pp 245-254. https://doi.org/10.5121/csit.2012.2226

26. Wang $\mathrm{CJ}$, Fang $\mathrm{H}$, Wang $\mathrm{C}$, et al. (2015) A novel initialization method for particle swarm optimization-based FCM in big biomedical data, pp 2942-2944

27. Silva Filho TM, Pimentel BA, Souza RMCR, Oliveira ALI (2015) Hybrid methods for fuzzy clustering based on fuzzy c-means and improved particle swarm optimization. Expert Syst Appl 42:6315-6328. https://doi.org/10.1016/j.eswa.2015.04.032

28. Niu Q, Huang X (2011) An improved fuzzy C-means clustering algorithm based on PSO. J Softw 6:873-879. https://doi. org/10.4304/jsw.6.5.873-879

29. Maulik U, Bandyopadhyay S (2000) Genetic algorithm-based clustering technique. Pattern Recognit 33:1455-1465. https:// doi.org/10.1016/S0031-3203(99)00137-5

30. Mekhmoukh A, Mokrani K (2015) Improved fuzzy C-means based particle swarm optimization (PSO) initialization and outlier rejection with level set methods for MR brain image segmentation. Comput Methods Programs Biomed 122:266-281. https://doi.org/10.1016/j.cmpb.2015.08.001

31. Liu J, Qiao S (2015) A image segmentation algorithm based on differential evolution particle swarm optimization fuzzy c-means clustering. Comput Sci Inf Syst 12:873-893. https:// doi.org/10.2298/CSIS141108031L

32. Das S, Konar A (2009) Automatic image pixel clustering with an improved differential evolution. Appl Soft Comput 9:226-236. https://doi.org/10.1016/j.asoc.2007.12.008

33. Maulik U (2009) Differential evolution based fuzzy clustering technique : application to satellite image segmentation, $\mathrm{pp}$. 362-368

34. Chaghari A, Balafar M (2018) Fuzzy clustering based on Forest optimization algorithm. J King Saud Univ - Comput Inf Sci 30:25-32. https://doi.org/10.1016/j.jksuci.2016.09.005

35. Ahmed MN, Yamany SM, Mohamed N et al (2002) A modified fuzzy c-means algorithm for bias field estimation and segmentation of MRI data. IEEE Trans Med Imaging 21:193-199. https ://doi.org/10.1109/42.996338

36. Chen S, Zhang D (2004) Robust image segmentation using FCM with spatial constraints based on new kernel-induced distance measure. IEEE Trans Syst Man Cybern Part B Cybern 34:1907-1916. https://doi.org/10.1109/TSMCB.2004.831165

37. Szilagyi' L, Benyol Z (2003) MR brain image segmentation using an enhanced fuzzy C-means algorithm. In: Proceedings of the 25th annual international conference of the IEEE EMBS Cancum, Mexico, pp 724-726

38. Cai W, Chen S, Zhang D (2007) Fast and robust fuzzy c-means clustering algorithms incorporating local information for image segmentation. Pattern Recognit 40:825-838. https:// doi.org/10.1016/j.patcog.2006.07.011

39. Lei T, Jia X, Zhang $Y$ et al (2018) Significantly fast and robust fuzzy C-Means clustering algorithm based on morphological reconstruction and membership filtering. IEEE Trans Fuzzy Syst 26:3027-3041. https://doi.org/10.1109/TFUZZ.2018.2796074

40. Satish R, Kumar PR (2020) Efficient method for segmentation of noisy and non-circular iris images using improved particle swarm optimisation-based MRFCM. IET Biom 9:78-90. https ://doi.org/10.1049/iet-bmt.2019.0026

41. Caselles V, Kimmel R, Sapiro G (1995) geodesic active contours. IEEE Intl Conf Comput Vis 22:694-699

42. Civicioglu P (2012) Transforming geocentric cartesian coordinates to geodetic coordinates by using differential search algorithm. Comput Geosci 46:229-247. https://doi.org/10.1016/j. cageo.2011.12.011

43. Liu B (2014) Composite differential search algorithm. J Appl Math. https://doi.org/10.1155/2014/294703 
44. Masek L, Kovesi P (2003) A biometric identification system based on iris patterns. The school of Computer Science and Software Engg, The University of Western Australia, Crawley

45. Fuentes-Hurtado F, Naranjo V, Diego-Mas JA, Alcañiz M (2019) A hybrid method for accurate iris segmentation on at-a-distance visible-wavelength images. EURASIP J. Image Video Process. 2019:75. https://doi.org/10.1186/s13640-019-0473-0
Publisher's Note Springer Nature remains neutral with regard to jurisdictional claims in published maps and institutional affiliations. 